\title{
The lung microbiome: the perfect culprit for COPD exacerbations?
}

\author{
Carine Gomez ${ }^{1}$ and Pascal Chanez ${ }^{2}$
}

Affiliations: ${ }^{1}$ Dept of Respiratory Diseases, Lung Transplant Team APHM, URMITE CNRS IRD UMR 6236, IHU Méditerranée Infection, Aix-Marseille University, Marseille, France. ${ }^{2}$ Dept of Respiratory Diseases APHM, INSERM CNRS U 1067, UMR7333, Aix-Marseille University, Marseille France.

Correspondence: Pascal Chanez, Dept of respiratory diseases. Hopital Nord 13015, Marseille, France. E-mail: pascal.chanezQuniv-amu.fr

0 @ERSpublications

Interaction between lung microbiota and inflammation is closely related to the pathogenesis of COPD exacerbation http://ow.ly/Z1B7o

Chronic obstructive pulmonary disease (COPD) is a heterogeneous group of diseases which affects more and more patients in Europe and represents an important challenge for the respiratory community. These airway diseases present different faces from a clinical, functional and pathophysiological point. So, we should better understand the underlying mechanisms contributing to the various phenotypes, which are probably the consequences of interactions between genes and environmental factors. Our current treatments are mainly symptom-driven and do not interfere with the natural history of the disease. The accelerated decline in lung function and the recurrence of severe exacerbations are important features representing most of the patient burden. Exacerbations are clearly defined at least from a clinical perspective, now, but still represent a challenging goal to be fully deciphered. A better understanding of the pathophysiological mechanisms of these acute events is required to optimise their management and their prevention.

It has been clearly established that exposure to microorganisms, such as viruses or bacteria, is related to the occurrence of exacerbations in COPD [1]. The remaining episodes without any causative factor may represent the limitations of the methods of detection of any pathogen. It is always challenging to relate the presence of a microorganism to a complex event such as a COPD exacerbation and direct accountability is sometimes difficult to establish. They are numerous reports questioning whether the role of pathogens is as a real culprit or as a bystander [2].

Since the 1980s, new microbial identification techniques have demonstrated that respiratory airways, long regarded as sterile, contain different pathogen communities, known as the lung microbiome [3]. Microbiomes, formerly called microbial flora, corresponds to all bacteria, viruses, fungi and other microorganisms in the human body. In healthy subjects, the total number of bacteria in the lung tissue has a density of 10-100 per 1000 human cells, which is relatively low compared with the gut flora [4]. Microbiomes play a crucial role in the development and integrity of the immune system. If certain bacteria are essential in the development of a healthy immune system, the modification of the lung microbiome can lead to diseases (figure 1) [5].

Compared with the abundant literature on the gut microbiome, the studies of lung microbiome or microbiota associated with the inception, loss of control and severity of chronic airway disorders are gradually leading to more reports in our respiratory journals [6].

In this issue of the European Respiratory Journal, WANG et al. [7] report specific changes in microbiota during COPD exacerbations and its association with the host inflammatory response thanks to data from a larger cohort with longitudinal 16S ribosomal RNA follow up. 467 sputum samples from 87 subjects with COPD were obtained at steady state, during exacerbation, and 2 and 6 weeks after recovery. The authors not

Received: Feb 042016 | Accepted: Feb 052016

Conflict of interest: Disclosures can be found alongside the online version of this article at erj.ersjournals.com

Copyright OERS 2016 
a)

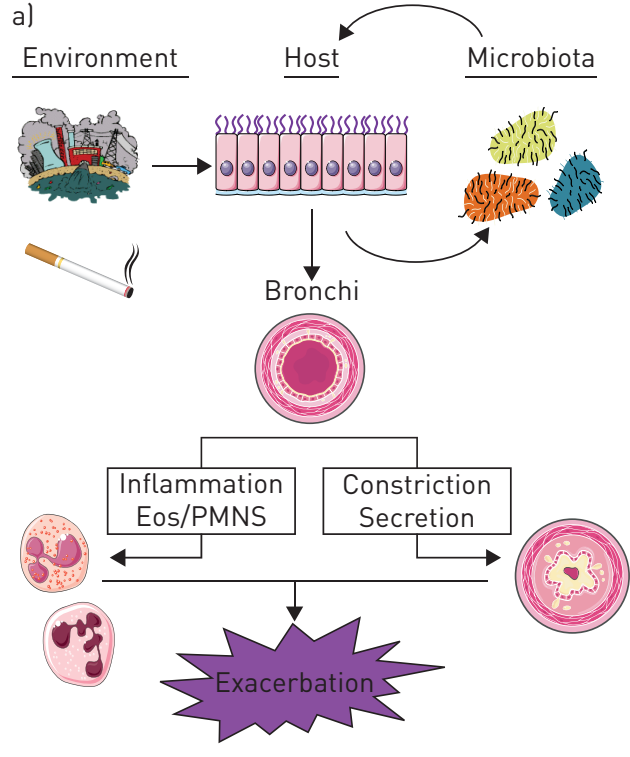

b)

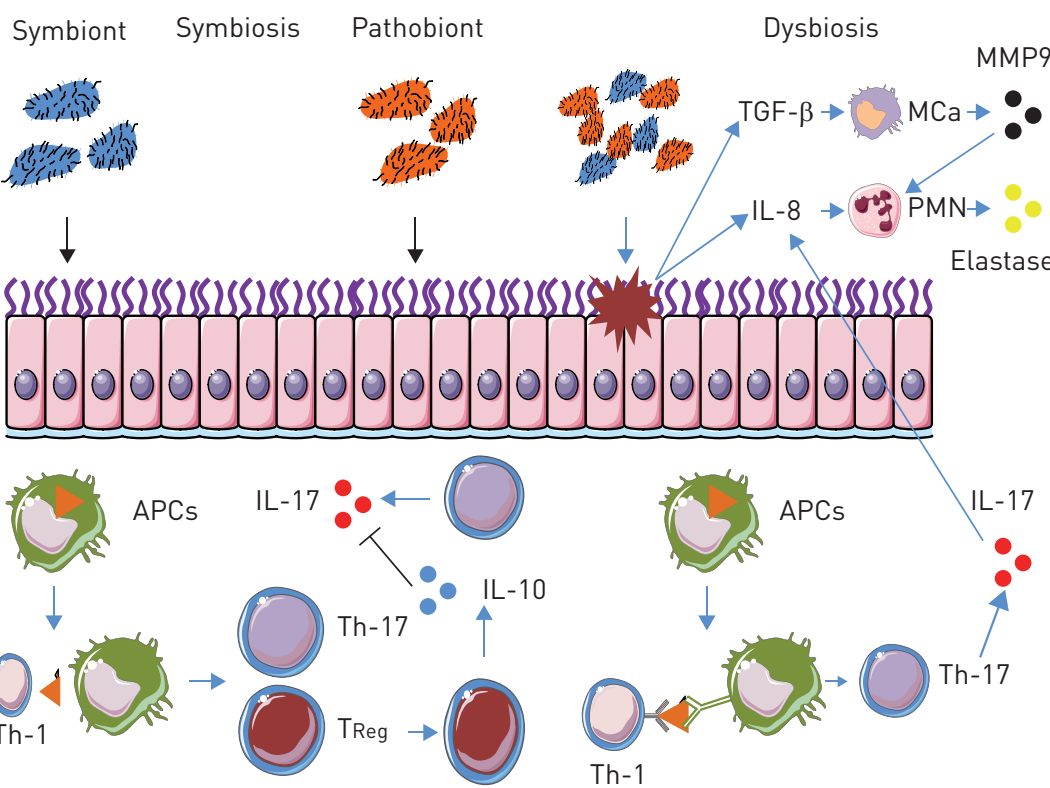

FIGURE 1 Exacerbations: the players. a) Interactions between the host and lung microbiota is a delicate balance. Deleterious factors affect innate immunity leading to lung microbiome changes resulting in inflammation and activation of resident cells resulting in exacerbation of chronic obstructive pulmonary disease. b) A healthy microbiota contains a balanced composition of symbionts and pathobionts which have the potential to induce inflammatory stimuli and cause imbalance of T-helper (Th) 17 and regulatory T-cells (Treg). Eos: eosinophils; TGF: transforming growth factor; IL: interleukin; MCa: macrophage; PMN: polymorphonuclear leukocytes; MMP: matrix metalloproteinases; APC: antigen presenting cell.

only showed microbiome shifts during exacerbations but also differences related to the exacerbation phenotypes described as "bacterial or eosinophilic". The major strengths of the present study are related to the high numbers of patients recruited and followed. It is an important contribution and a success by analysing such a huge number of samples and to relate the findings to predefined phenotypes, biomarkers and treatments. In such a study, one may always question the potential for false contamination [8] and the real clinical significance of this big data analysis. A specific attention to current versus ex-smokers in the present study is missing and may provide interesting differences to better understand and manage patients with COPD exacerbations. Smoking is the most important risk factor for COPD, but only about $20 \%$ of smokers develop the disease and smoking cessation does not reverse its evolution. Therefore other factors are needed and the impact of smoking on the respiratory microbiome is important. Cigarette smoke exposure alters bronchial epithelium resulting in various changes in the release of inflammatory mediators involved in macrophages and neutrophil recruitment and activation [9]. The expression of toll like receptor (TLR) 2 and TLR4 is modified contributing to a potential increased susceptibility for bacterial infections [10, 11].

Exacerbations in COPD patients are variable in frequency and are perhaps a risk factor for fast decline of the pulmonary function. Therefore, strategies aiming to a future risk assessment are crucial to develop. Whereas neutrophilic inflammation is associated with COPD exacerbations, the existence of a phenotype associated with eosinophilic inflammation has been extensively described [12]. The inflammatory patterns have been related to the responses to therapies used to treat exacerbations [13, 14]. The most frequent phenotypes of COPD exacerbations, i.e.: "bacterial" and "eosinophilic" [15], have been revisited by WANG et al. [7]. Considering the "bacterial phenotype", they reported an increase in Proteobacteria when in "eosinophilic phenotype" an increase in Firmicutes was noted. In addition to differences in the microbiome of these two major phenotypes, response to treatment usually used during the exacerbations was also different: the bacterial phenotype answered more to antibiotics and the eosinophilic phenotype to corticosteroid. Several reports show the major impact of treatments used on the lung microbiome. Antibiotics are supposed to clear infection and restore an initial "safe" microbiome; however, this can lead to a niche for outgrowth of opportunistic bacteria or fungi [16]. It was found that antibiotic use affects the lung microbiome and leads to an increase of alpha diversity and Proteobacteria and decreased of Firmicutes in subjects treated (with or without steroids). Similarly to the work of HuANG et al. [17], the present study highlights a change in microbiome induced by corticosteroids alone with an increase, at the genus level, of Haemophilus and Moraxella spp. The impact of inhaled treatment on airway microbiota has already been mentioned. Pragman et al. [18] have thus noted that the microbiota of COPD patients treated with inhaled corticosteroid and bronchodilator were different from untreated patients demonstrating that 
the microbiome is not only dependent on the disease but also on the type of medication usage. These findings should be kept in mind considering the relationships between inhaled corticosteroids exposure and the occurence of "pneumonias" in patients suffering from COPD [19].

The keystone-pathogen hypothesis holds that certain low-abundance microbial pathogens can orchestrate inflammatory disease by remodelling a normally benign microbiota into a dysbiotic one. This is an interesting view which may be sustained by the present findings but will require more data to be fully integrated into the potential inception and future risks for patients with a COPD [20].

Overall, the research, including the present study, investigating the relationships between the lung microbiome and chronic airway disorders is paramount to better understanding and treatment of patients suffering from different phenotypes of COPD.

\section{References}

1 Papi A, Bellettato CM, Braccioni F, et al. Infections and airway inflammation in chronic obstructive pulmonary disease severe exacerbations. Am J Respir Crit Care Med 2006; 173: 1114-1121.

2 Alikhan MM, Lee FE-H. Understanding nontypeable Haemophilus influenzae and chronic obstructive pulmonary disease. Curr Opin Pulm Med 2014; 20: 159-164.

3 Charlson ES, Bittinger K, Haas AR, et al. Topographical continuity of bacterial populations in the healthy human respiratory tract. Am J Respir Crit Care Med 2011; 184: 957-963.

4 Turnbaugh PJ, Ley RE, Hamady M, et al. The human microbiome project. Nature 2007; 449: 804-810.

5 Gollwitzer ES, Saglani S, Trompette A, et al. Lung microbiota promotes tolerance to allergens in neonates via PD-L1. Nat Med 2014; 20: 642-647.

6 Sze MA, Dimitriu PA, Hayashi S, et al. The lung tissue microbiome in chronic obstructive pulmonary disease. Am J Respir Crit Care Med 2012; 185: 1073-1080.

7 Wang Z, Bafadhel M, Haldar K, et al. Lung microbiome dynamics in COPD exacerbations. Eur Respir J 2016; 47: 1082-1092.

8 Salter SJ, Cox MJ, Turek EM, et al. Reagent and laboratory contamination can critically impact sequence-based microbiome analyses. BMC Biol 2014; 12: 87.

9 Garmendia J, Morey P, Bengoechea JA. Impact of cigarette smoke exposure on host-bacterial pathogen interactions. Eur Respir J 2012; 39: 467-477.

10 Knobloch J, Schild K, Jungck D, et al. The T-helper cell type 1 immune response to gram-negative bacterial infections is impaired in COPD. Am J Respir Crit Care Med 2011; 183: 204-214.

11 Zuo L, Lucas K, Fortuna CA, et al. Molecular Regulation of Toll-like Receptors in Asthma and COPD. Front Physiol 2015; 6: 312.

12 Saetta M, Di Stefano A, Maestrelli P, et al. Airway eosinophilia in chronic bronchitis during exacerbations. Am J Respir Crit Care Med 1994; 150: 1646-1652.

13 Ram FSF, Rodriguez-Roisin R, Granados-Navarrete A, et al. Antibiotics for exacerbations of chronic obstructive pulmonary disease. Cochrane Database Syst Rev 2006; CD004403.

14 Brightling CE, Monteiro W, Ward R, et al. Sputum eosinophilia and short-term response to prednisolone in chronic obstructive pulmonary disease: a randomised controlled trial. Lancet Lond Engl 2000; 356: 1480-1485.

15 Bafadhel M, McKenna S, Terry S, et al. Acute exacerbations of chronic obstructive pulmonary disease: identification of biologic clusters and their biomarkers. Am J Respir Crit Care Med 2011; 184: 662-671.

16 Sullivan A, Edlund C, Nord CE. Effect of antimicrobial agents on the ecological balance of human microflora. Lancet Infect Dis 2001; 1: 101-114.

17 Huang YJ, Sethi S, Murphy T, et al. Airway microbiome dynamics in exacerbations of chronic obstructive pulmonary disease. J Clin Microbiol 2014; 52: 2813-2823.

18 Pragman AA, Kim HB, Reilly CS, et al. The lung microbiome in moderate and severe chronic obstructive pulmonary disease. PloS One 2012; 7: e47305.

19 Kew KM, Seniukovich A. Inhaled steroids and risk of pneumonia for chronic obstructive pulmonary disease. Cochrane Database Syst Rev 2014; 3: CD010115.

20 Hajishengallis G, Darveau RP, Curtis MA. The keystone-pathogen hypothesis. Nat Rev Microbiol 2012; 10: $717-725$. 